\title{
Retrieval of contextual information from memory'
}

\author{
SAUL STERNBERG \\ BELL TELEPHONE LABORATORIES
}

When Ss name the item that follows a test item in a short recently memorized list, the ir mean reaction-time $(\overline{\mathrm{RT}})$ increases linearly with list length. The linearity and slope of the function, and the effect of the test item's serial position, imply that the test item is located in the memorized list by an internal self-terminating scanning process whose average rate is about four items/sec.

In previous experiments Ss judging whether a test item was contained in a short memorized list produced $\overline{\mathrm{RT}} \mathbf{s}$ that increased as a function of list length (Sternberg, $1966,1967)$. Various findings, including the linearity and slope of the function, led to a theory of high-speed internal scanning in which the test item is compared successively to the items in memory at an average rate between 25 and 30 items/sec. Each comparison results in either a match or a mismatch; a positive response is made if there has been a match, and a negative response otherwise. $\overline{\mathrm{RT}}$ increased with list length at the same rate for positive and negative responses, indicating that instead of being self-terminating (positive response initiated as soon as a match occurs), the process has the remarkable property of being exhaustive (even when a match has occurred, scanning continues through the entire list).

The high-speed scanning process was revealed when Ss had to ascertain the presence or absence of an item in a memorized list. In contrast, the test item in the present experiment had to be located in the list, since its context was to furnish the required response (the name of the item that followed the test item).

Method

Ss were six undergraduates who had served for $2 \mathrm{hr}$. in another reaction-time experiment. On each trial s different random digits, $3 \leq s \leq 7$, appeared singly at a fixed locus for $0.54 \mathrm{sec}$. each. ${ }^{2}$ The listlength, $s$, varied at random from trial to trial. There followed a $2.5 \mathrm{sec}$. delay during which silent rehearsal was encouraged, a 1.3 sec. warning signal, and then a test stimulus, randomly selected from among the first s-1 items in the list. The correct response was the spoken name of the item that followed the test item in the list. A voice-operated relay was used to measure the RT (time from onset of test stimulus to occurrence of vocal response). Between the response and the presentation of the next list, 2.6 sec. elapsed. In each of three sessions, $\mathrm{S}$ had 15 practice trials and 180 test trials. Feedback (every 30 trials), scoring system, and payoffs were designed to encourage $S$ to respond as rapidly as possible while maintaining a low error-rate.

Results

Data from Sessions 2 and 3 were combined for each S; means over the six Ss are shown in Fig. $1 .^{3}$
Length function: For correct responses, linear regression (Fig. 1A) accounts for $99.0 \%$ of the variation of $\overline{\mathrm{RT}}$ with list length (the "length function"). The slope of the fitted line is $124.0 \pm 15.3 \mathrm{msec}$./item; its zerointercept is $294.7 \pm 31.5 \mathrm{msec}$. Lines for individual Ss differ significantly in slope $(p=.005)$; the population distribution of slopes has an estimated SD of 34.5 msec./ item. 4

Position functions. For lists of all lengths there was a pure primacy effect: $\overline{\mathrm{RT}}$ increased with serial position of the test item (Fig. 1B; $p=.04$ ). The mean slope of the five position functions is positive for every $S$, with a mean over $\mathrm{Ss}$ of $92.4 \pm 34.2 \mathrm{msec}$./item, but exhibits much more $(p=.03)$ inter $-S$ variation than the lengthfunction slope (estimated population SD is $79.9 \mathrm{msec}$./ item). These differences between $\mathrm{Ss}$ were stable over sessions. Figure 2 shows two extreme examples of sets of position functions from Ss whose length-function slopes are approximately equal.

Errors: Errors increased sharply with s(Fig.1A), the overall rate being $8.2 \%$. They included naming of test items $(12 \%)$, other incorrect items in the series $(69 \%)$, and items (digits) not in the series (19\%). Errors were most frequent for test items in later serial positions. Discussion

The following theory is proposed to account for these findings. In retrieval of contextual information the test item must first be located in the memorized list. This is achieved by a self-terminating process of scanning to locate: the test item is compared successively to the items in memory until a match occurs. When the next item is to be named, the search is followed by a shift from test item to adjacent response item. The mean time from the beginning of one comparison to the beginning of the next, and the mean time to shift, are affected by

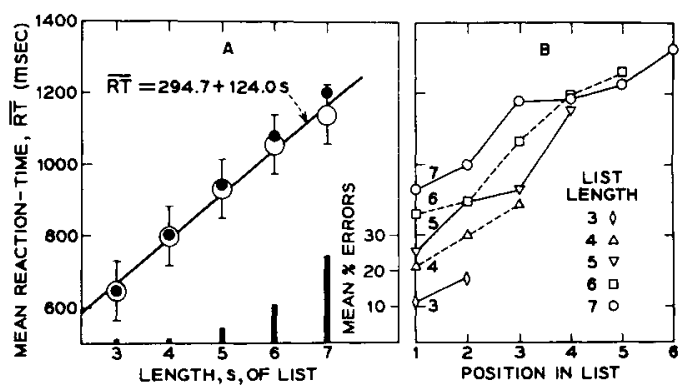

Fig. 1. A. Effect of list length, $s$, on accuracy of response and $\overline{\mathbf{R T}}$. Percent errors (bars), $\overline{\mathbf{R T}}$ of correct responses (open circles, about 400 observations each) with estimates of $\pm \sigma$ and leastsquares line, and $\overline{\mathrm{RT}}$ of all responses (filled circles). ${ }^{4} \mathrm{~B}$. Relation between $\overline{\mathrm{RT}}$ of correct responses and serial position of the test item in lists of five lengths. 


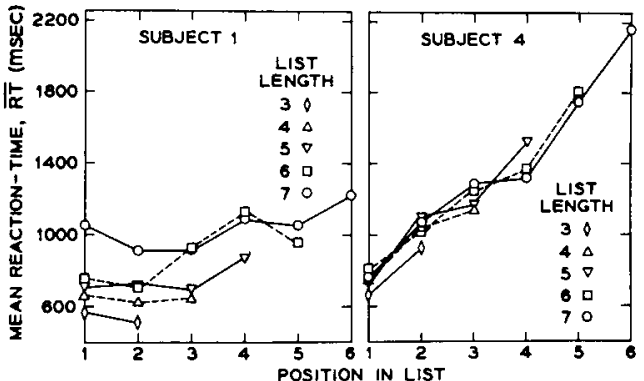

Fig. 2. Contrasting sets of position functions in lists of five lengths, one set relatively flat and separated, the other steep and, in general, superimposed. Mean slopes are 22 and $240 \mathrm{msec}$./item, for $\mathrm{S1}$ and S4, respectively. $\overline{\mathrm{RT}}$ scale differs from that of Fig. 1. Length functions for $\mathrm{S1}$ and $\mathrm{S4}$ (not shown) have almost equal slopes (126 and $146 \mathrm{msec}$./item, respectively).

neither list length nor serial position.

If scanning invariably began at the first position in the list and proceeded serially, the position functions would be linear, increasing, and superimposed; if the test item appeared in positions 1 to $s-1$ with equal frequency, as in the present experiment, the mean number of comparisons would be $s / 2$. An alternative is "circular" scanning with a random starting point. (Random starting might arise, for example, if the presentation of the test item interrupted an ongoing cyclic rehearsal process, and scanning then began at the serial position where rehearsal happened to end.) The position functions would then be flat and separated and the mean number of comparisons would be $(s+1) / 2.5$

With both strategies the length function would be linear (Fig. 1A) with a slope equal to half the mean time from the beginning of one comparison to the beginning of the next. Hence, two Ss who scanned at the same rate but differed in starting strategy would produce similar length functions but radically different sets of position functions (Fig. 2). 6 Data from the six Ss appear to reflect mixtures, in different proportions, of the two starting strategies. ${ }^{7}$

A length-function slope of 124 msec./item implies, for a self-terminating search, a rate of $248 \mathrm{msec}$./item. Hence, the rate of "scanning to locate" is about 4 items/ sec., one-seventh the rate of "scanning for presence." Even when an item is known to be in a memorized list, access to It appears to be far from direct-a conclusion similar to the one Hoffding reached from other considerations (Rock, 1962).

Why does locating an item in a list call for a process that is so much slower than the one used to ascertain its presence? The answer to this question may provide some insight into the puzzling exhaustiveness of the high-speed process. Suppose a retrieval system in which (a) the occurrence of a match, but not the location of the item that produced it, is entered in a register if and when it takes place, (b) a separate operation of checking the register is required for detecting whether a match occurred, and (c) the checking operation is slow, and cannot occur concurrently with the comparison operation. In such a system, self-terminating search would entail a long interruption after each comparison for checking the register.

When the required response depends only on presence or absence, sufficient information could be obtained by checking the register just once, after performing comparisons throughout the entire list; although this would increase the mean number of comparisons on trials requiring positive responses, it could nonetheless yield shorter $\overline{\mathrm{RT}} \mathrm{s}$ than self-terminating search. This would account for the combination of exhaustiveness and high speed in "scanning for presence." But when the response entails locating an item in the list, the high-speed process could not be used: for location of the item that produced a match, the register would have to be checked after each comparison. Scanning would then be slower; and, since further comparisons after a match is detected would be superfluous, it could be self-terminating.

\section{References}

Rock, I. A neglected aspect of the problem of recall: The Höffding function. In J. M. Scher (Ed.), Theories of the mind. New York: Free Press, 1962.

Stemberg, S. High-speed scanning in human memory. Science, 1966, $153,652-654$.

Stemberg, S. Two operations in character recognition: Some evidence from reaction-time measurements. Percept.\& Psychophys., 1967, 2, 45-53.

\section{Notes}

1. Supported in part by NSF grant GB-1172 to the University of Pennsylvania. I thank M. and R. Teghtsoonian, C. S. Harris, J. Krauskopf, and H. Savin for helpful discussions.

2. For three $S s$ the rate was changed to $0.37 \mathrm{sec}$./digit in Session 3. Effects on $\overline{\mathrm{RT}}$ were small and nonsignificant. data for the two rates were pooled.

3. $\overline{\mathrm{RT}} \mathrm{S}$ in Session 1 are not given in detail because of the high error-rate $(13.7 \%$ overall, $39.4 \%$ when $s=7$ ) but were similar to those reported: for correct responses the function $\overline{\mathrm{RT}}=368.7+$ $123.6 \mathrm{~s}$ accounts for $95.5 \%$ of the variation of $\overline{\mathrm{RT}}$ with $\mathrm{s}$; the mean position-function slope is $89.1 \mathrm{msec}$./ item.

4. Where quantities are stated in the form $x \pm y, y$ is the $S E$ of $x$. SEs and SD estimates depend on variance components derived from analyses of variance in which individual differences in mean and slope were treated as "random effects."

5. For the process with a random starting point these statements are, in general, only approximate. They are exact if the test item may be compared to the last item although they will never match, and if the mean time between comparisons of the test item to the last and first items does not differ from the mean time between other pairs of successive comparisons.

6. An alternative explanation of flat, separated position functions might be a strategy of exhaustive scanning. One argument against this explanation is the behavior of the variance of the RTs for a particular length and position, averaged over positions. Approximately equal for $\mathrm{S} 1$ and $\mathrm{S4}$ when $\mathrm{s}=3$, this quantity grows with $\mathrm{s}$ twice as rapidly for $\mathrm{S} 1$ as for $\mathbf{S 4}$. $\mathbf{S 1}$ 's high variability could arise from a random starting strategy, but not from exhaustiveness. 7. The existence of even one $S$ with approximately superimposed position functions suggests that RT in this situation (and thus scanning rate and shift time) are not affected by either memory load per se, or stimulus or response uncertainty, all of which increase with s. 